\title{
Seeking a science of instruction
}

\author{
Richard E. Mayer
}

Received: 1 September 2009/Accepted: 8 September 2009/Published online: 20 October 2009

(C) The Author(s) 2009. This article is published with open access at Springerlink.com

The design of instruction should be guided by a research-based theory of how instruction works - that is, a theory of how instructional methods affect learning processes. This is the central premise in the science of instruction (Clark and Mayer 2008; Mayer 2009). One of our field's most influential and most studied instructional theories is cognitive load theory (Sweller 1999, 2005), which is the subject of a thought-provoking critique by Ton de Jong (this issue). In this commentary, I explore the strengths and limitations of de Jong's paper and explore three exemplary issues in more detail.

\section{Strengths and limitations}

On the positive side, de Jong's paper offers a detailed, comprehensive, and systematic review of cognitive load theory by a knowledgeable author who has contributed much to our field. The author provides a service by raising many relevant criticisms of aspects of the theory (e.g., calling for clarifying key concepts), the research methodology (e.g., calling for better measures of cognitive load), and the supporting evidence (e.g., noting inconsistencies in the findings). Our field could benefit from a thoughtful attempt to resolve some of the criticisms raised in de Jong's review.

On the negative side, this paper provides mainly a catalogue of criticisms of cognitive load theory without offering much of a direction of where to go from here. In a sense, it is a tribute to cognitive load theory that a leading instructional researcher would devote so much effort to pinpointing the many shortcomings he sees in the theory and its supporting evidence. In order to offer a constructive critique that has the potential to move our field forward, the next step is to move from criticizing to building. I attempt to take this kind of a constructive approach in the remainder of this commentary by trying to reconcile three exemplary issues raised in de Jong's fine review—realistic situations, boundary conditions, and triarchic theory.

R. E. Mayer $(\square)$

University of California, Santa Barbara CA, USA

e-mail: mayer@psych.ucsb.edu 


\section{Towards a resolution of the issue of realistic situations}

In the section on "The external validity of research results," de Jong questions whether the results of laboratory studies with short lessons apply to more authentic learning situations in real schools. This is certainly a legitimate concern, which we have repeatedly recognized in our own research reports. However, it is widely recognized that educational research is strengthened by multiple research methods (Shavelson and Towne 2005), so it is useful to conduct both lab-based and school-based studies. Each approach has its own strengths and weaknesses, so together they can help us build a more complete science of instruction. It should be noted that several of the papers by Sweller and colleagues cited in de Jong's review involve trainees in industrial training programs, thereby offering some evidence of external validity. Similarly, although most of our work on the modality principle has been conducted in labs with short lessons, I am pleased to report that on occasion, some researchers have had the courage to investigate the modality principle in real school settings (Harskamp et al. 2007). The call to conduct research in lab and school venues should not be seen as a challenge to instructional theory but rather as a way of broadening it.

\section{Towards a resolution of the issue of boundary conditions}

In the section entitled, "The external validity of research results," de Jong provides a selective review of research findings on the modality principle (Low and Sweller 2005; Mayer 2009) in which the main critique is that the modality effect is not found in all studies. For example, the modality effect tends to be strongest when the material is fastpaced (i.e., presented at a fast pace for the learner) and under system control (i.e., not paced by the learner). I refer to these kinds of qualifications as boundary conditions (Mayer 2009). Far from being a challenge to the underlying theory (which in my case is the cognitive theory of multimedia learning), the discovery of boundary conditions can help to verify the predictions of the theory, to sharpen the theory, and to better present practical implications. In the case of the modality principle, the cognitive theory of multimedia learning and cognitive load theory predict the kinds of boundary conditions that have been observed in the literature.

In short, the discovery of boundary conditions should not be seen as inconsistent with the cognitive theory of multimedia learning or cognitive load theory, but rather as a way of testing (and in this case, verifying) the predictions of the theory. On the theoretical level, the search for boundary conditions should be seen as a way to help instructional theory continue to develop, rather than as a way of shooting it down. On the practical level, research-based principles such as the modality principle should be implemented in ways that are consistent with the underlying theory rather than being seen as immutable prescriptions that must be followed in all situations.

\section{Towards a resolution of the issue of three kinds of cognitive load}

In the section on "The three kinds of cognitive load revisited," de Jong raises many questions about the clarity of the definitions and measures of intrinsic, extraneous, and germane cognitive load. In particular, he raises the potentially productive question of whether cognitive load theory is mainly about the different kinds of demands on working memory or about different kinds of cognitive processing during learning. 
Let me try to briefly summarize how the cognitive theory of multimedia learning addresses the issue of three kinds of cognitive load-which I call the triarchic theory-in hopes of bringing some reconciliation. In our work (Mayer 2009), we have found it useful to distinguish among three kinds of demands on the learner's limited cognitive capacityextraneous processing, which is cognitive processing that does not serve the instructional objective and is caused by unhelpful instructional design or learner characteristics; essential processing, which is cognitive processing required to mentally represent the presented material in working memory and is caused by the complexity of the material; and generative processing, which is cognitive processing required to make sense of the material and is caused by the learner's motivation.

According to the theory, meaningful learning depends on appropriate cognitive processing during learning, including selecting relevant words and pictures for further processing, organizing the selected material into coherent mental representations, and integrating the mental representations with each other and with relevant prior knowledge activated from long-term memory. Extraneous processing involves none of these three processes; essential processing involves selecting and some initial organizing; and generative processing involves further organizing and integrating. This conceptualization has helped us discover several research-based principles of multimedia learning (Mayer 2009) and has been at least partially validated in a study by DeLeeuw and Mayer (2008) showing that extraneous, essential, and generative processing are tapped by different assessment instruments. Again, having to explain the three kinds of cognitive load in terms of underlying cognitive processing is not a shortcoming but rather a way of improving the usefulness of instructional theories.

In summary, the construction of a research-based science of instruction is a collaborative effort that benefits from constructive criticism. A critique that does nothing but bash a theory is too negative and a critique that does nothing but back a theory is too positive, but a critique that seeks to build a reconciliation between a theory and its criticisms provides a the way to move our field forward. A critique such as de Jong's is useful to the extent that it helps us build the most useful instructional theory possible.

Open Access This article is distributed under the terms of the Creative Commons Attribution Noncommercial License which permits any noncommercial use, distribution, and reproduction in any medium, provided the original author(s) and source are credited.

\section{References}

Clark, R. C., \& Mayer, R. E. (2008). e-Learning and the science of instruction. San Francisco: Pfeiffer.

DeLeeuw, K. E., \& Mayer, R. E. (2008). A comparison of three measures of cognitive load: Evidence for separable measures of intrinsic, extraneous, and germane load. Journal of Educational Psychology, $100,223-234$.

Harskamp, E., Mayer, R. E., Suhre, C., \& Jansma, J. (2007). Does the modality principle for multimedia learning apply to science classrooms? Learning and Instruction, 17, 465-477.

Low, R., \& Sweller, J. (2005). The modality principle in multimedia learning. In R. E. Mayer (Ed.), The Cambridge handbook of multimedia learning (pp. 147-158). New York: Cambridge University Press.

Mayer, R. E. (2009). Multimedia learning (2nd ed.). New York: Cambridge University Press.

Shavelson, R. J., \& Towne, L. (Eds.). (2005). Scientific research in education. Washington, DC: National Academy Press.

Sweller, J. (1999). Instructional design in technical areas. Camberwell, Australia: ACER Press.

Sweller, J. (2005). Implications of cognitive load theory for multimedia learning. In R. E. Mayer (Ed.), The Cambridge handbook of multimedia learning (pp. 19-30). New York: Cambridge University Press. 